\title{
Hypermethylation of miRNA-589 promoter leads to upregulation of HDAC5 which promotes malignancy in non-small cell lung cancer
}

\author{
CHANGHONG LIU ${ }^{1}$, DESHENG LV ${ }^{1}$, MO LI ${ }^{1}$, XUEFEI ZHANG ${ }^{1}$, \\ GE SUN ${ }^{1}$, YU BAI ${ }^{1}$ and DONGMIN CHANG ${ }^{2}$ \\ ${ }^{1}$ Department of Thoracic Surgery, The Second Affiliated Hospital of Dalian Medical University, \\ Dalian, Liaoning 116021; ${ }^{2}$ Department of Surgical Oncology, The First Affiliated Hospital \\ of Xi'an Jiaotong University, Xi'an, Shaanxi 710061, P.R. China
}

Received January 25, 2017; Accepted March 20, 2017

DOI: 10.3892/ijo.2017.3967

\begin{abstract}
Histone deacetylases (HDACs) are crucial for regulating chromatin activity, which plays a critical role in cell proliferation, differentiation, and apoptosis of various cancers. Therefore, HDAC inhibitors have been applied as effective therapeutic agents for cancer treatment. However, the expression profiles and regulatory mechanisms of histone deacetylases in lung cancer are not well understood. In the present study, aberrant high levels of HDAC5 were observed in non-small cell lung cancer (NSCLC) and further analysis indicated a negative relationship between HDAC5 and a tumor suppressor, miR-589-5p, in NSCLC specimens. Consistently, miR-589-5p reduced the expression of HDAC5 by targeting the 3'UTR of HDAC5 mRNA in NSCLC cells. Considering the loss of miR-589-5p in NSCLC, the methylation status of the miR-589 gene promoter was examined. The hypermethylation of the miR-589 gene promoter was more significant in NSCLC cells compared with lung epithelial cells, and methylation inhibition by 5-aza-2-deoxycytidine (5-Aza-dC) decreased HDAC5 expression. Furthermore, several downstream gene clusters of HDAC5 were studied in the present investigation. As a result, miR-589-5p/HDAC5 pathway was found to regulate a number of cell cycle and epithelial-mesenchymal transition (EMT)-related genes in NSCLC cells. In vitro and in vivo phenotype experiments revealed a critical role of miR-589-5p/HDAC5 pathway in the migration, invasion, and tumorigenicity of NSCLC cells. These findings demonstrate a novel mechanism for deregulation of HDAC5 in NSCLC and
\end{abstract}

Correspondence to: Professor Dongmin Chang, Department of Surgical Oncology, The First Affiliated Hospital of Xi'an Jiaotong University, 227 Yantaxi Road, Xi'an, Shaanxi 710061, P.R. China E-mail: changdongmin1234@163.com

Key words: non-small cell lung cancer, HDAC5, miR-589-5p, hypermethylation suggest that miR-589-5p/HDAC5 pathway may represent a new prognostic biomarker and therapeutic target against NSCLC.

\section{Introduction}

Lung cancer has become one of the major causes for cancerrelated deaths in the last few years, most cases of which are non-small cell lung cancer (NSCLC). Although, in the past decade, target therapy such as epidermal growth factor receptor (EGFR) antagonist has been applied in NSCLC treatment, mutations of target genes and drug resistance damaged the curative effect of target therapy (1). As a novel therapeutic strategy, epigenetics approach impairs the drug resistance resulted from cancer heterogeneity by regulating the cluster of tumor suppression genes. Among these epigenetics approaches, hypomethylating agents and histone deacetylase (HDAC) inhibitors have been widely used in clinical treatment of various tumors including lung cancer (2). However, the tumorrelated genes and signal pathways targeted by epigenetic therapy are largely unknown.

Histone deacetylases (HDACs) are the critical enzymes that catalyze the removal of acetyl groups from the lysine residues within histones. Therefore, HDAC family displays a determinant role in transcriptional regulation by controlling chromatin condensation. Based on their structure and homology, human HDACs have been classified into four classes (classes I, II, III and IV) (3). HDAC5 belongs to class II HDACs (4-10) which play a crucial role in tumorigenesis $(4,5)$. In the present study, overexpression of HDAC5 was found to be closely associated with activation of tumor drivers and inhibition of tumor suppressors in NSCLC cells. Consistently, numerous studies showed that HDACs are aberrantly expressed in various cancer types and then change the epigenetic status which blocks the expressions of tumor suppressors (6). Consequently, abnormal expressions of HDACs promote cell proliferation, invasion and even resistance to multi-drugs which contribute to a poor prognosis in different cancers $(7,8)$. Although some members of HDACs have been well investigated in several cancer types, the molecular mechanisms for the functions and deregulation of HDAC5 in human NSCLC remain unclear. 
Earlier research found that overexpression of miR-589 partially reversed the TGF- $\beta 1$ induced epithelial-mesenchymal transition (EMT) change of human peritoneal mesothelial cells (9). Although, as an important microRNA, miR-589 were rarely studied in tumor progression until recently. Li et al reported that miR-589 was significantly downregulated in cisplatin-resistant A549, and transfection with miR-589 resulted in an increased sensitivity to cisplatin (10). A later study showed that miR-589-5p could downregulate the stemness characteristics of $\mathrm{CD} 90^{+}$cancer stem cells (CSCs) of HCC in part by silencing MAP3K8 (11). Our findings revealed that the loss of miR-589-5p by hypermethylation accelerated NSCLC development through increasing the expression of HDAC5.

In the present study, higher expression of HDAC5 accompanied with decreased miR-589-5p was observed in NSCLC compared with normal tissues. Furthermore, miR-589-5p significantly reduced HDAC5 expression by targeting the 3'UTR of HDAC5 mRNA. Promoter hypermethylation of the miR-589 gene was also observed in NSCLC cells, but not in lung epithelial cells, which caused the loss of miR-589-5p. Consequently, aberrant regulation of miR-589-5p/HDAC5 pathway promoted the cell cycle and EMT-related gene clusters and then conferred the aggressiveness of NSCLC cells.

\section{Materials and methods}

Cell culture and drug treatment. Human NSCLC cell lines A549, H1299, and human lung epithelial cell line BEAS-2B were purchased from the American Type Culture Collection (ATCC). A549 and H1299 cells were cultured in Dulbecco's modified Eagle's medium (DMEM, Corning) supplemented with $10 \%$ fetal bovine serum (FBS, Gibco), $100 \mathrm{U} / \mathrm{ml}$ penicillin, and $100 \mathrm{U} / \mathrm{ml}$ streptomycin. BEAS-2B cells were cultured in RPMI-1640 (Corning) supplemented with same additive. All cells were grown at $37^{\circ} \mathrm{C}$ in $5 \% \mathrm{CO}_{2}$. A549 and H1299 cell lines were treated with 5-Aza-dC (Sigma) at a final concentration of $4 \mu \mathrm{M}$.

Ethics approval. All procedures performed in studies involving human participants were in accordance with the ethical standards of the institutional and/or national research committee and with the 1964 Helsinki declaration and its later amendments or comparable ethical standards.

mRNA and miRNA quantification. TRIzol reagent was used to extract total RNA. miRNA was amplified using the TaqMan MicroRNA Reverse Transcription kit (Applied Biosystems). Mature miR-589-5p transcript levels were measured with TaqMan MicroRNA assay primers (Applied Biosystems) and the mRNA levels of indicated genes were measured using SYBR Premix Ex Taq ${ }^{\mathrm{TM}}$ (Takara). miRNA or mRNA levels were quantified with the ABI PRISM 7500 real-time PCR system (Applied Biosystems).

DNA sodium bisulfite conversion. Genomic DNA was extracted from indicated cells by phenol-chloroform technique. Bisulfite conversion was performed as described (12). Specific primers for converted promoter region were used to generate PCR product. Forward, TTTTGGAGTTTTTTTTGGTTTTT; reverse, CAAAAACAAAACCAAAATCA. PCR products were cloned in pUCm-T followed by sequencing with $\mathrm{T} 7$ primer.

QPCR array. The high-throughput profiling of gene clusters were analyzed by The ExProfile ${ }^{\mathrm{TM}}$ Gene qPCR array (GeneCopoeia) according to the manufacturer's instructions. In 96-well plate, there are 50 pairs of qPCR primers and 12-wells of controls which are used to monitor the efficiency of the entire experimental process (from reverse transcription to qPCR reaction). A cDNA pool, containing reverse transcript products from total RNA of the indicated cells, was used as the $\mathrm{qPCR}$ validation template.

CCK-8 assay. Cells in logarithmic growth were plated and transfected. After indicated time of culture, CCK-8 (Cell Counting Kit-8) (Dojindo Molecular Technologies, MD, USA) was added, and the OD450 was measured using an automatic plate reader.

Cell cycle analysis. Cells were treated with the indicated reagents and fixed in $70 \%$ ethanol overnight. Fixed cells were treated with $100 \mathrm{mg} / \mathrm{ml}$ RNaseA (Roche) before addition of $50 \mathrm{mg} / \mathrm{ml}$ PI (Sigma) and analyzed by FACSCalibur flow cytometer.

EdU incorporation assay. The indicated cells were seeded in 96-well plates. DNA synthesis was assessed using the Cell-Light EdU (5-ethynyl-2'-deoxyuridine) DNA Cell Proliferation kit (RiboBio) according to the manufacturer's instructions. Images of the cells were captured with a fluorescence microscope. ImageJ software was used to count the fluorescent points.

Wound healing assay. Transfected cells were grown to $80 \%$ confluence in 6-well tissue culture plates and wounded with a sterile tip to remove cells by perpendicular linear scrapes. After that, the cells were cultured for $24 \mathrm{~h}$. The wound was photographed and quantified immediately after wounding and again $24 \mathrm{~h}$ later.

Cell invasion assays. Cell invasion were assessed in Boyden chambers with Matrigel according to the manufacturer's protocol (Invitrogen). First, an 8-mm-porosity polycarbonate membrane was covered with $200 \mu \mathrm{l}$ of serum-free medium containing $1 \times 10^{5}$ cells per well. The plates were then incubated with $10 \% \mathrm{FBS}$ medium for $48 \mathrm{~h}$ at $37^{\circ} \mathrm{C}$ in a $5 \% \mathrm{CO}_{2}$ incubator. The invasion cells on the bottom surface of the filter were fixed, stained, and counted using optical microscopy.

Colony formation in soft agar. For colony formation experiment, cells were suspended in $0.3 \%$ agar and seeded into 6-well plate pre-coated with $1.0 \mathrm{ml}$ of $0.6 \%$ agar. Medium was changed every 5 days for three weeks. The number and size of colonies were examined and data were obtained by analyzing with ImageJ software.

Nude mouse xenograft assay. Twenty-five female BALB/c nude mice were injected with A549 cells with stable overexpression of miR-589-5p or HDAC5. All of the mice were randomly 
divided into five groups ( $\mathrm{n}=5$ of each group), and each received a subcutaneous injection of a viable cell suspension mixture $\left(5 \times 10^{6}\right)$ containing $90 \%$ of A549 cells with indicated transfection. When the tumors could be palpated, tumor size was measured using calipers every three days. All of the mice were sacrificed on the fifth week after injection, and the individual tumors were weighed.

Immunohistochemistry. The study was performed with the approval of the Ethics Committee of The First Affiliated Hospital of Xi'an Jiaotong University and the Second of Dalian Medical University. NSCLC tissues were collected by needle biopsy or surgery from 190 patients at The First Affiliated Hospital of Xi'an Jiaotong University (Xi'an, China) and The Second of Dalian Medical University (Dalian, China) from 2008 to 2010. All specimens were fixed in $10 \%$ neutral formalin, embedded in paraffin and cut into $4-\mu \mathrm{m}$ sections for immunohistochemical staining. The EnVision ${ }^{\mathrm{TM}}$ two-step method was used (Dako, Hamburg, Germany), as well as the following antibody: antibody against HDAC5. To estimate the score for each slide, at least 10 individual fields at $200 \mathrm{X}$ were chosen, and 100 cancer cells were counted in each field. The immunostaining intensity was divided into four grades: 0 , no expression; 1 , mildly positive; 2 , moderately positive; and 3 , markedly positive. The proportion of positive-staining cells was divided into five grades: $0,<10 \% ; 1,11-25 \% ; 2,26-50 \%$; $3,51-75 \%$; and $4,>75 \%$. The staining results were assessed and confirmed by two independent investigators blinded to the clinical data. The percentage of positivity of the tumor cells and the staining intensities were then multiplied in order to generate the IHC score, and graded as 0-6, low expression; 7-12, high expression. Cases with a discrepancy in scores were discussed to obtain a consensus.

Statistical analysis. All statistical analyses were carried out using the SPSS 17.0 statistical software. Experimental data were presented as mean \pm SD from at least three independent experiments. The data were analyzed by Student's t-test. Statistical analysis to evaluate correlation was performed using Pearson's correlation analysis. A p $<0.05$ was considered statistically significant.

\section{Results}

A significant negative correlation between miR-589-5p and HDAC5 was found in NSCLC specimens. To characterize the expression level of HDAC5 in NSCLC, immunohistochemical analysis of tumor tissues from NSCLC patients was performed. As demonstrated in Fig. 1A, different protein levels of HDAC5 were observed in the NSCLC tissues from different patients. Further study showed that high expression of HDAC5 was positively correlated with lymph node metastasis, differentiation status and TNM stage $(\mathrm{P}<0.05)$ but not with gender, age, tumor size and histological type $(\mathrm{P}>0.05)$ (Table I). Also, the 5-year overall survival (OS) rate of HDAC5 high expression group was significantly lower than that of HDAC5 low expression group (13.41 vs 26.85\%, $\mathrm{P}=0.0061$ ) (Fig. 1B). Ectopic expression of HDAC5 mRNA was found in NSCLC but not in normal lung tissues (Fig. 1C). Consistent with previous study, miR-589-5p, known as a tumor suppressor gene, was down-
Table I. Correlation of the expression of HDAC5 with clinicopathological features in non-small cell lung cancer tissues.

\begin{tabular}{|c|c|c|c|c|c|c|}
\hline & & & & $\begin{array}{l}\text { HDAC } \\
\text { press }\end{array}$ & & \\
\hline & Total & - & + & ++ & +++ & P-value \\
\hline & 190 & 24 & 69 & 72 & 25 & \\
\hline Gender & & & & & & 0.7352 \\
\hline Male & 132 & 18 & 51 & 45 & 18 & \\
\hline Female & 58 & 6 & 18 & 27 & 7 & \\
\hline Age (years) & & & & & & 0.6278 \\
\hline$<57$ & 88 & 15 & 29 & 34 & 10 & \\
\hline$\geq 57$ & 102 & 9 & 40 & 38 & 15 & \\
\hline Tumor size $(\mathrm{cm})$ & & & & & & 0.1027 \\
\hline$\leq 3$ & 76 & 10 & 39 & 19 & 8 & \\
\hline$>3$ & 114 & 14 & 30 & 53 & 17 & \\
\hline Histological type & & & & & & 0.2856 \\
\hline $\begin{array}{l}\text { Squamous cell } \\
\text { carcinoma }\end{array}$ & 63 & 8 & 25 & 21 & 9 & \\
\hline Adenocarcinoma & 106 & 14 & 36 & 44 & 12 & \\
\hline Mixed/other & 21 & 2 & 8 & 7 & 4 & \\
\hline Lymph node metast & asis & & & & & $0.0262^{\mathrm{a}}$ \\
\hline No & 84 & 14 & 42 & 21 & 7 & \\
\hline Yes & 106 & 10 & 27 & 51 & 18 & \\
\hline Differentiation statu & & & & & & $0.0085^{\mathrm{a}}$ \\
\hline Well & 48 & 13 & 25 & 7 & 3 & \\
\hline Moderate & 85 & 7 & 36 & 34 & 8 & \\
\hline Poor & 57 & 4 & 8 & 31 & 14 & \\
\hline TNM stage & & & & & & $0.0011^{\mathrm{a}}$ \\
\hline $\mathrm{I} / \mathrm{II}$ & 147 & 21 & 58 & 49 & 8 & \\
\hline III/IV & 43 & 3 & 11 & 23 & 17 & \\
\hline
\end{tabular}

${ }^{\mathrm{a}} \mathrm{P}<0.05$.

regulated in NSCLC compared to normal tissues (Fig. 1D). Notably, a statistically negative correlation between HDAC5 mRNA and miR-589-5p was confirmed in NSCLC specimens (Fig. 1E). The above findings reveal that overexpression of HDAC5 found in NSCLC indicates a poor prognosis, and may be associated with loss of miR-589-5p.

miR-589-5p targets the 3'UTR of HDAC5 and then reduces HDAC5 expression in NSCLC cells. To verify HDAC5 as a target of miR-589-5p, software TargetScan was applied to predict putative interaction and a possible binding site within the 3'UTR of HDAC5 was found to hybridize with miR-589-5p (Fig. 2A). Furthermore, the expressions of miR-589-5p and HDAC5 in lung epithelial or NSCLC cells were examined. Upregulation of HDAC5 accompanied with decreased miR-589-5p was observed in NSCLC cells compared with lung epithelial cells (Fig. 2B-E). The expressions of HDAC5 at 
A

HDAC5 expression level
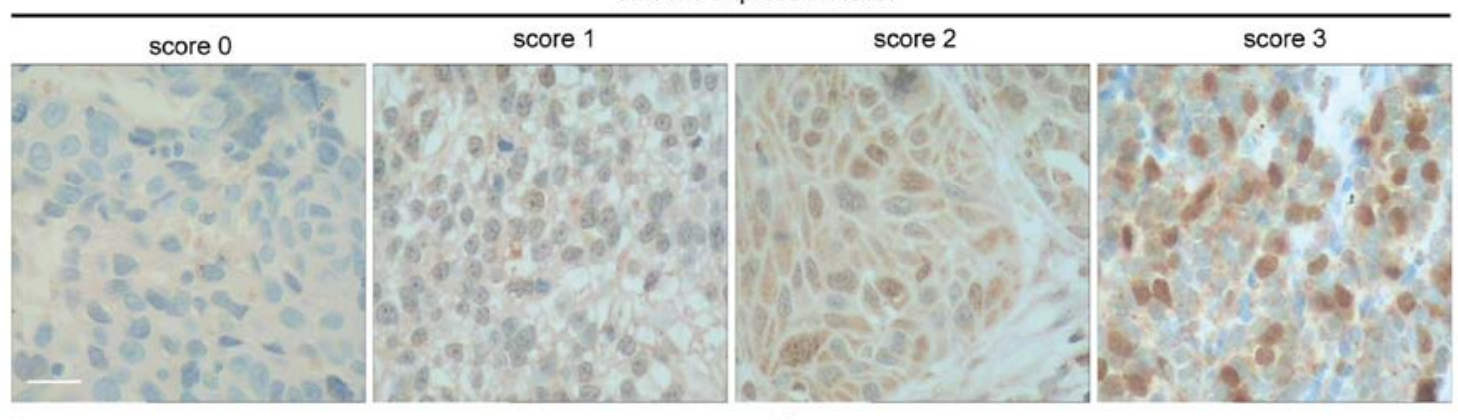

B

C
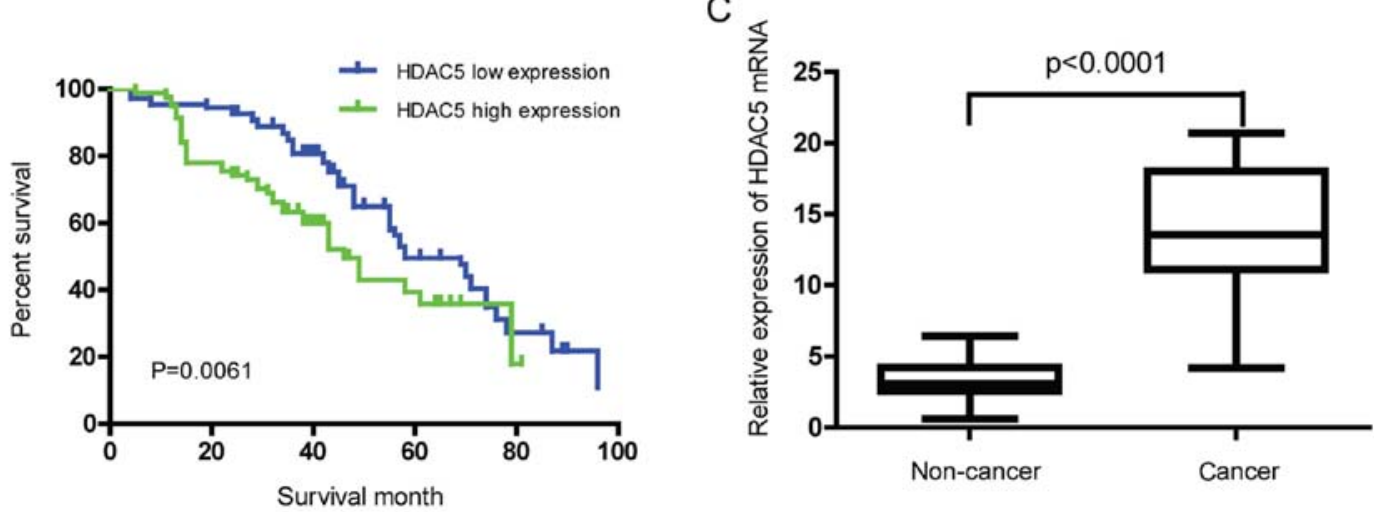

$\mathrm{D}$
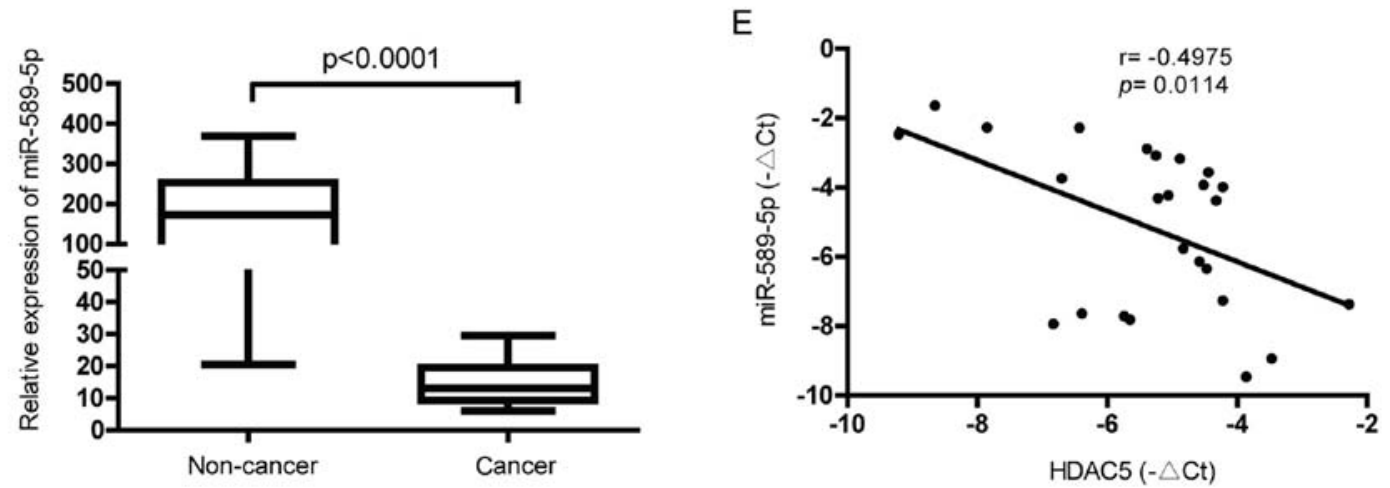

Figure 1. A significant negative correlation between miR-589-5p and HDAC5 was found in NSCLC specimens. (A) The representative immunohistochemical results of HDAC5 protein in NSCLC tissues (scale bar, $50 \mu \mathrm{m}$ ). (B) Kaplan-Meier survival curves for NSCLC patients divided by the levels of HDAC5. (C) Relative mRNA levels of HDAC5 in NSCLC tissues compared with normal tissues. (D) Relative expression of miR-589-5p in NSCLC tissues compared with normal tissues. (E) miR-589-5p and HDAC5 mRNA expression levels negatively correlated in 25 clinical NSCLC samples. Statistical analysis to evaluate correlation was carried out by Pearson's correlation analysis.

both mRNA and protein level were attenuated in NSCLC cells transfected with wild-type miR-589-5p, but not with the mutant (Fig. 2F and G). Knockdown of miR-589-5p in lung epithelial cells using an anti-miRNA inhibitor specific for miR-589-5p resulted in a significant upregulation of HDAC5 (Fig. $2 \mathrm{H}$ and I). To determine whether HDAC5 is directly targeted by miR-589-5p in NSCLC, HDAC5 3'UTR fragment containing a wild-type or mutant binding site was cloned into luciferase reporter vector and the inhibitory effect of miR-589-5p was measured by luciferase activity. It was found that the luciferase signal of wild-3'UTR was reactively decreased by miR-589-5p, but the mutant was not (Fig. 2J). Consistently, only the luciferase reporter with intact 3'UTR was responsive to miR-589-5p inhibitor (Fig. 2K). Hence, these data indicate that miR-589-5p negatively regulates HDAC5 expression via targeting the 3'UTR of HDAC5 mRNA.
Promoter hypermethylation of the miR-589 gene decreases the level of miR-589-5p and promotes HDAC5 expression. miR-589 is an intronic microRNA which is located at 7p22.1 and within intron 3 of the F-Box and leucine rich repeat protein 18 (FBXL18) gene, and they are in the same transcriptional direction (Fig. 3A). Further analysis showed that the expression patterns of miR-589 and its host gene, FBXL18, in NSCLC cell lines or after 5-Aza-dC treatment seemed to be similar, which suggested that they may share the same promoter (Fig. 3B-D). In order to characterize the DNA methylation profile of the FBXL18/miR-589 gene promoter, DNA bisulfate conversion coupled to sequencing was performed in NSCLC and lung epithelial cells. A CpG island was predicted using UCSC genome browser and focused on (Fig. 3A). High DNA methylation levels of the $\mathrm{CpG}$ island, with $86.4 \%$ of methylated CpGs in A549 cells and $77.4 \%$ in H1299 cells, 
A

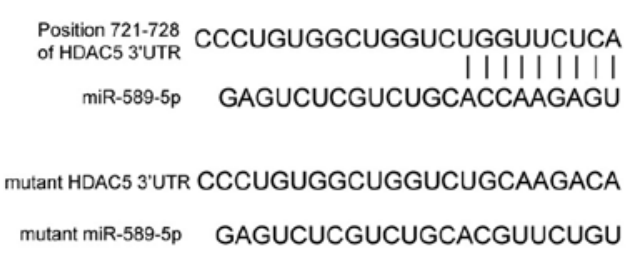

D

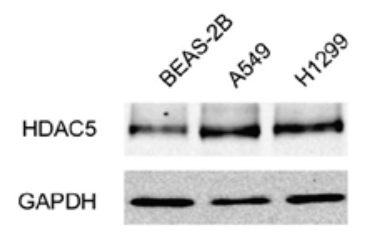

G

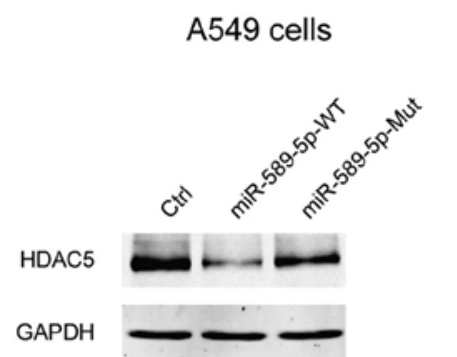

B

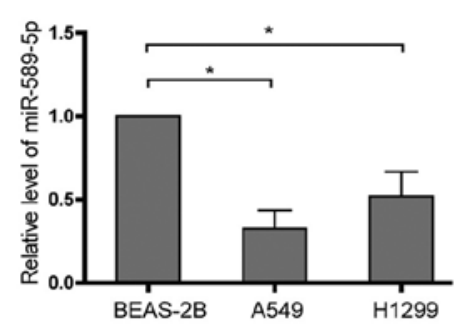

E

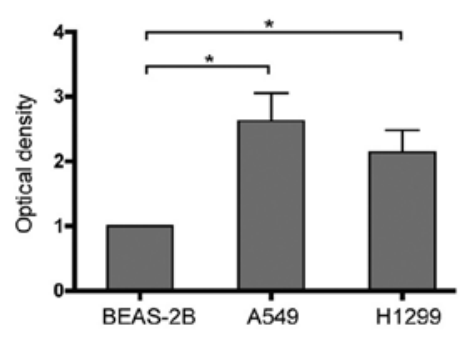

$\mathrm{H}$

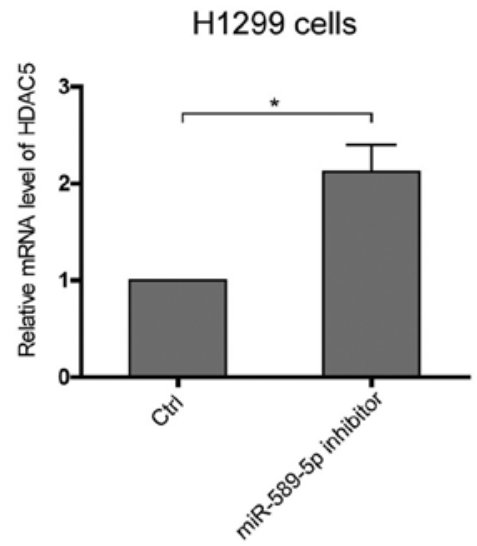

C

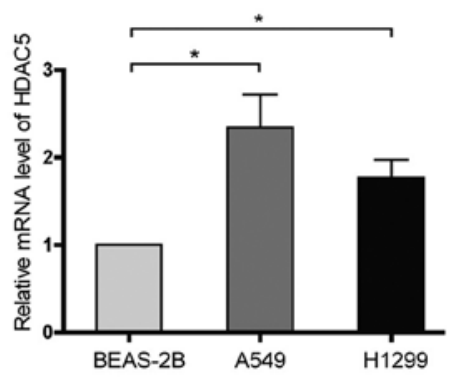

$\mathrm{F}$

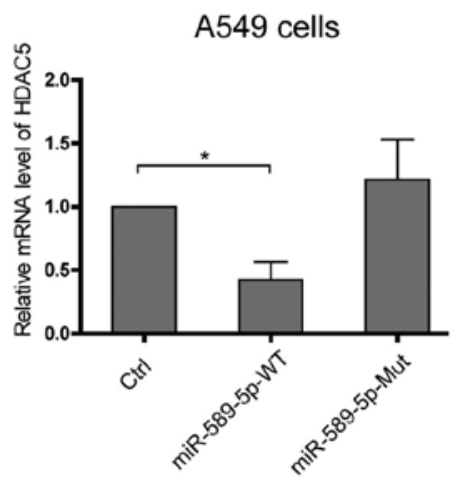

I

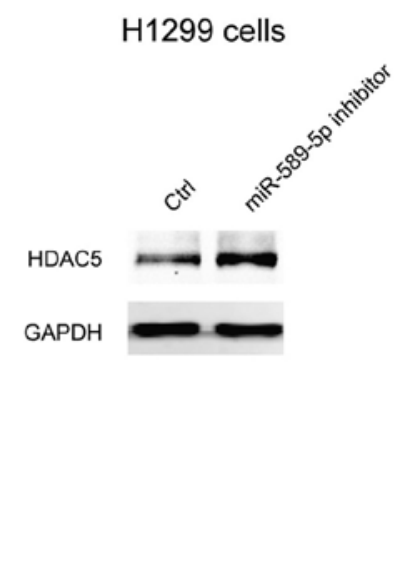

J

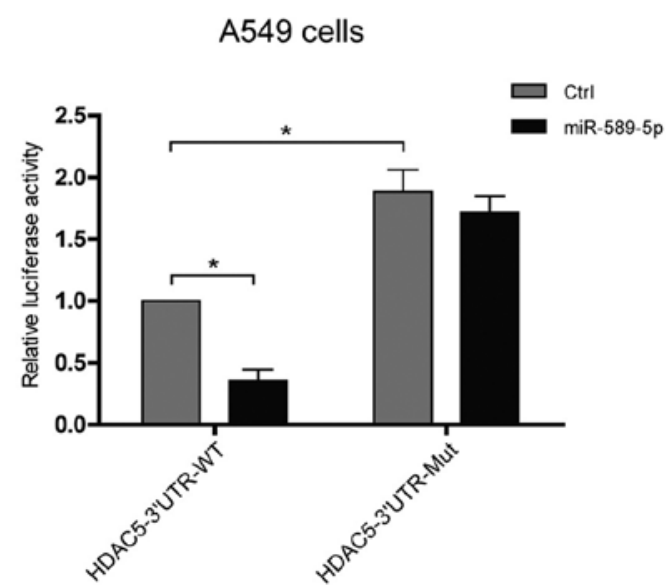

K
H1299 cells

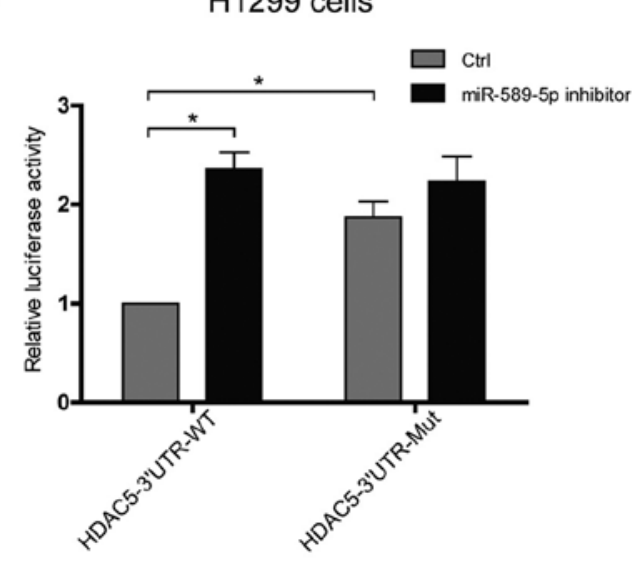

Figure 2. miR-589-5p targets the 3'UTR of HDAC5 and then reduces HDAC5 expression in NSCLC cells. (A) The putative human HDAC5-3'UTR fragments containing wild-type or mutant miR-589-5p binding sequence was inserted into the luciferase report vector pmirGLO downstream. (B-D) The different levels of miR-589-5p (B), HDAC5 mRNA (C), and HDAC5 protein (D) were measured by qPCR and western blotting in two NSCLC cell lines, A549 and H1299, and a lung epithelial cell line, BEAS-2B. (E) Quantitative analysis for (D). (F and G) The mRNA (F) and protein levels (G) of HDAC5 were measured by qPCR and western blotting in A549 cells transfected with wild or mutant miR-589-5p. ${ }^{*} \mathrm{P}<0.05$. (H and I) The mRNA (H) and protein levels (I) of HDAC5 were measured by qPCR and western blotting in H1299 cells transfected with miR-589-5p inhibitor or not. " $\mathrm{P}<0.05$. (J) Luciferase assay of A549 cells co-transfected with the luciferase constructs containing the wild or mutant HDAC5-3'UTR as well as miR-589-5p mimics or scrambled oligonucleotides as the negative control. ${ }^{*} \mathrm{P}<0.05$. (K) Luciferase assay of H1299 cells co-transfected with the luciferase constructs containing the wild-type or mutant HDAC5-3'UTR as well as miR-589-5p inhibitor or scrambled oligonucleotides as the negative control. ${ }^{*} \mathrm{P}<0.05$. 
A $\operatorname{chr} 7$ (p22.1)
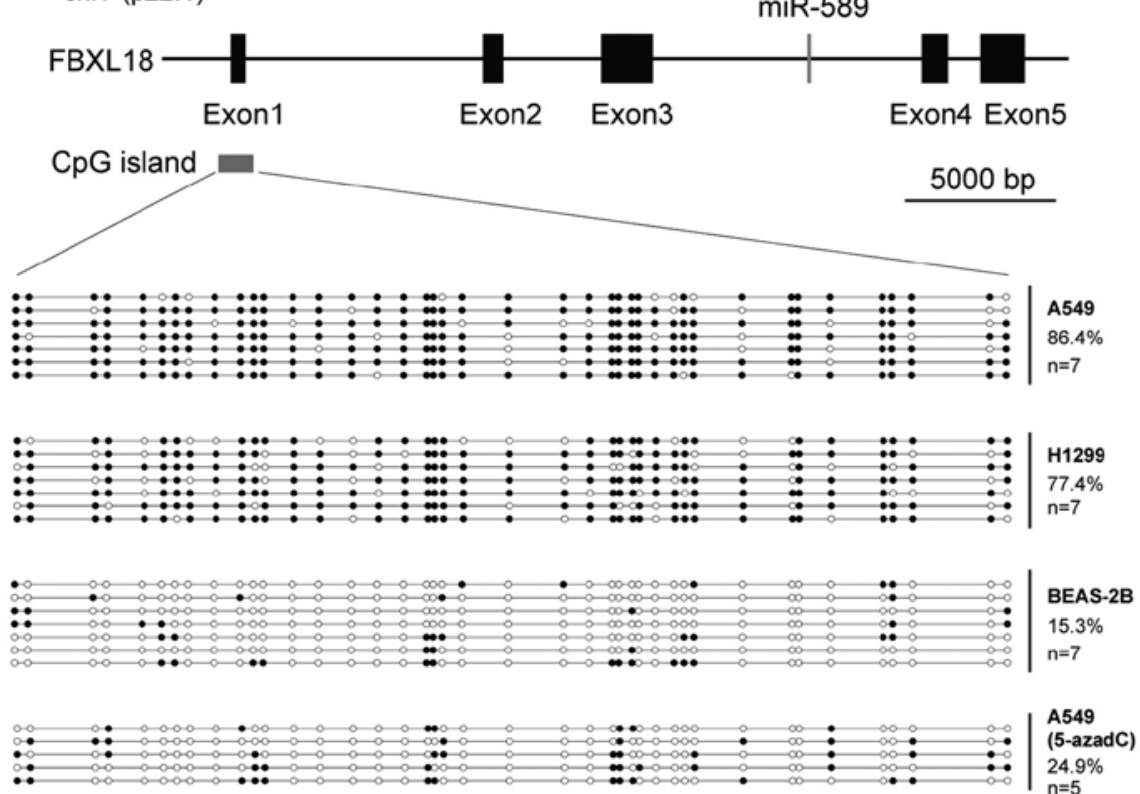

D

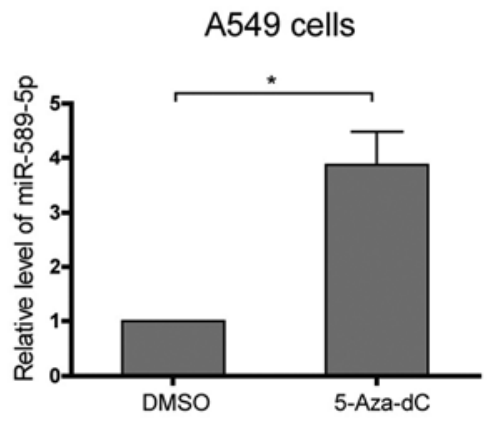

G

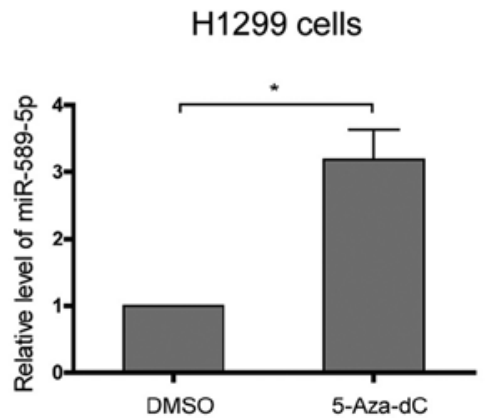

$E$

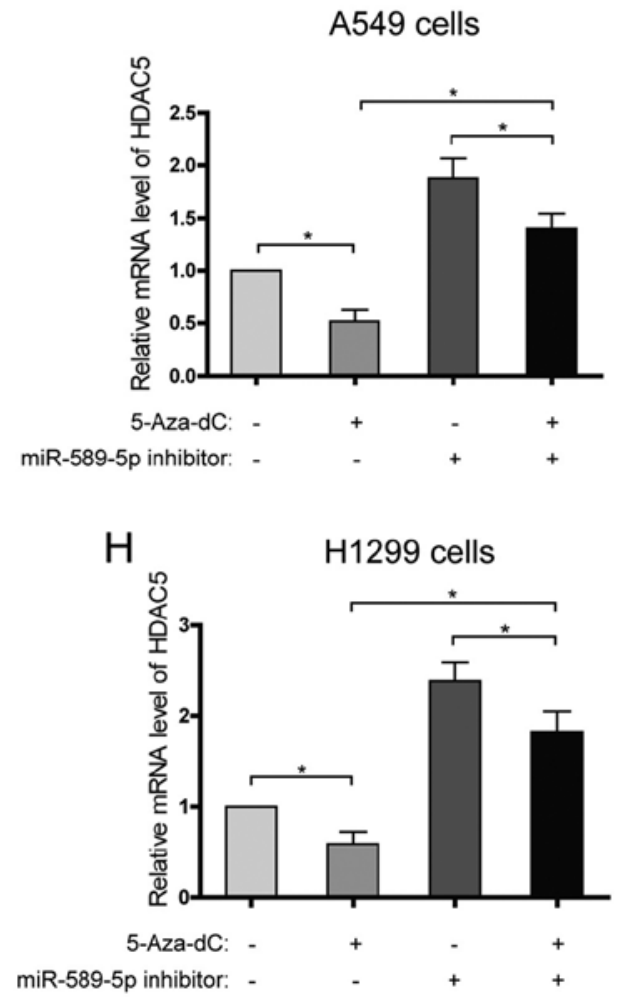

B

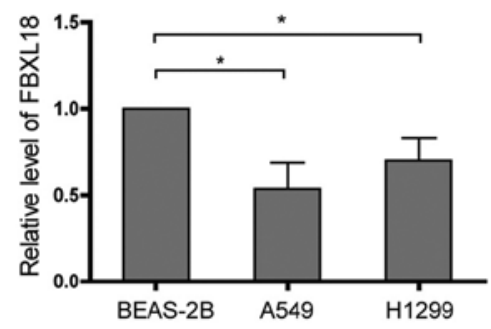

C

A549 cells

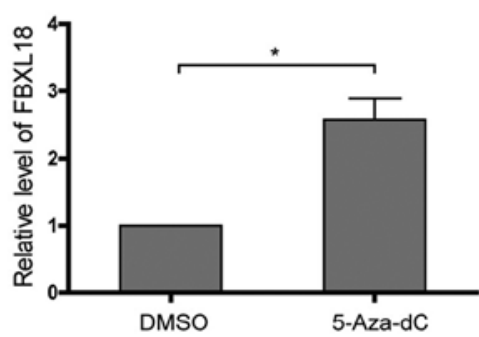

$\mathrm{F}$

A549 cells

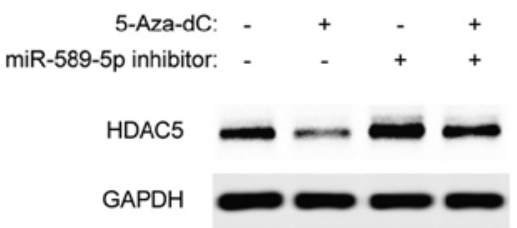

I

H1299 cells

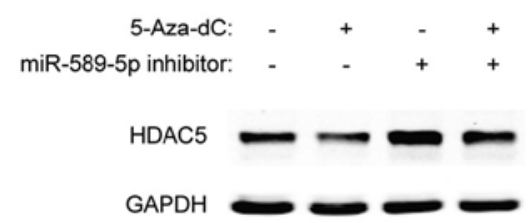

Figure 3. Promoter hypermethylation of the miR-589 gene decreases the level of miR-589-5p and promotes HDAC5 expression. (A) The hypermethylation levels of the FBXL18/miR-589 gene promoter in A549, H1299, BEAS-2B, and 5-Aza-dC treated A549 cells were determined by bisulphite DNA sequencing showing independent clones from each group. (B) The relative levels of FBXL18 were measured by qPCR in two NSCLC cell lines, A549 and H1299, and a lung epithelial cell line, BEAS-2B. "P<0.05. (C) Relative level of FBXL18 in A549 cells after 5-Aza-dC treatment. "P<0.05. (D) Relative level of miR-589-5p in A549 cells after 5-Aza-dC treatment. "P<0.05. (E and F) The mRNA (E) and protein (F) level of HDAC5 were measured by qPCR and western blotting in A549 cells co-treated with 5-Aza-dC and miR-589-5p inhibitor. " $\mathrm{P}<0.05$. (G) Relative level of miR-589-5p in H1299 cells after 5-Aza-dC treatment. "P<0.05. (H and I) The mRNA $(\mathrm{H})$ and protein (I) level of HDAC5 were measured by qPCR and western blotting in H1299 cells co-treated with 5-Aza-dC and miR-589-5p inhibitor. ${ }^{*} \mathrm{P}<0.05$.

were detected with DNA bisulfate conversion (Fig. 3A). In contrast, low DNA methylation level, with $15.3 \%$ of methylated CpGs, was found in the lung epithelial BEAS-2B cells
(Fig. 3A). To confirm that the promoter hypermethylation of the miR-589 gene enhances the transcriptional repression of miR-589-5p and then increases HDAC5 expression, NSCLC 
A

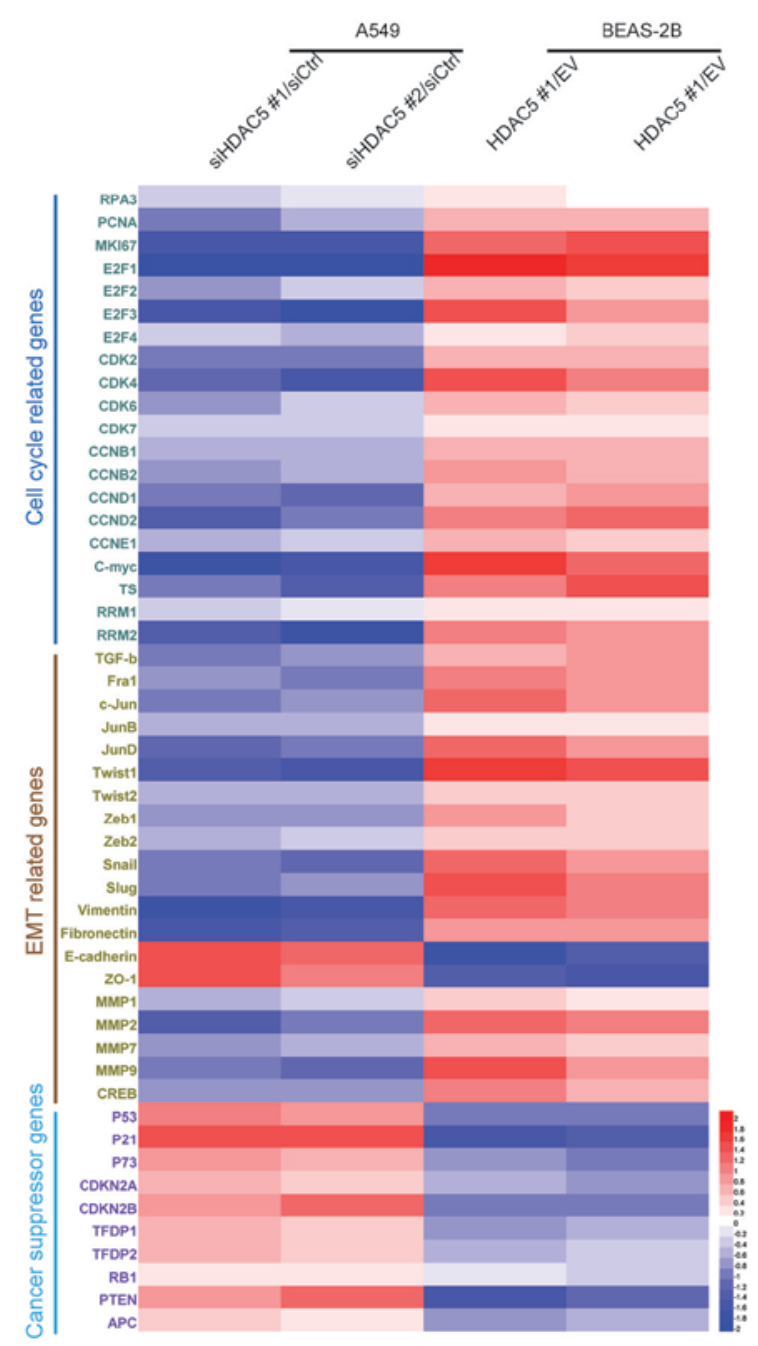

B

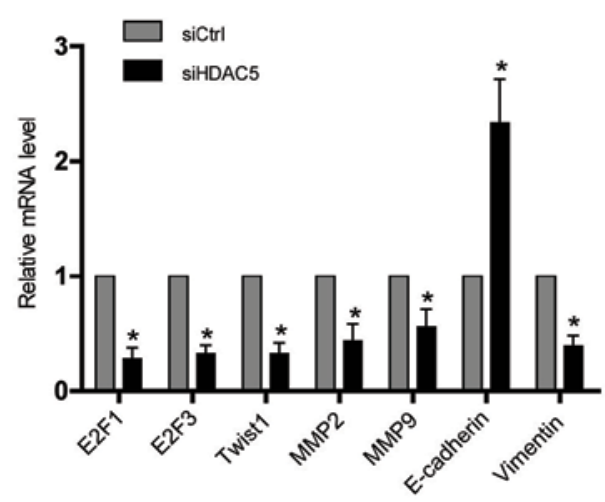

D

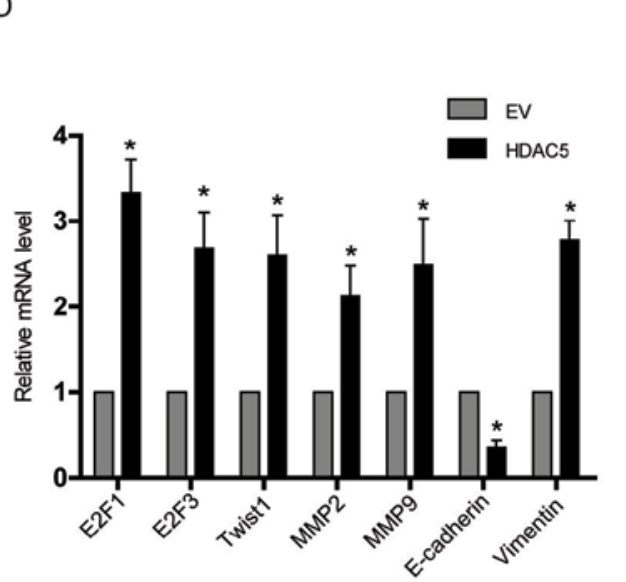

C

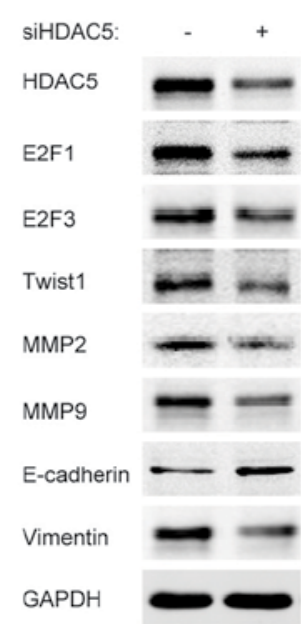

$E$

EV HDAC5

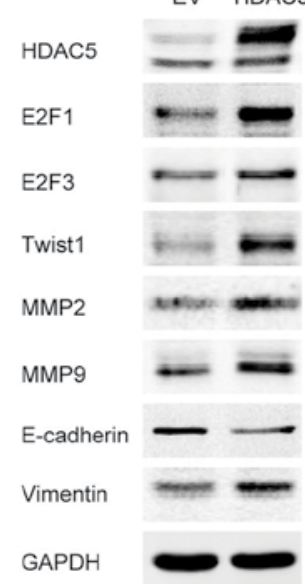

G

$\mathrm{F}$

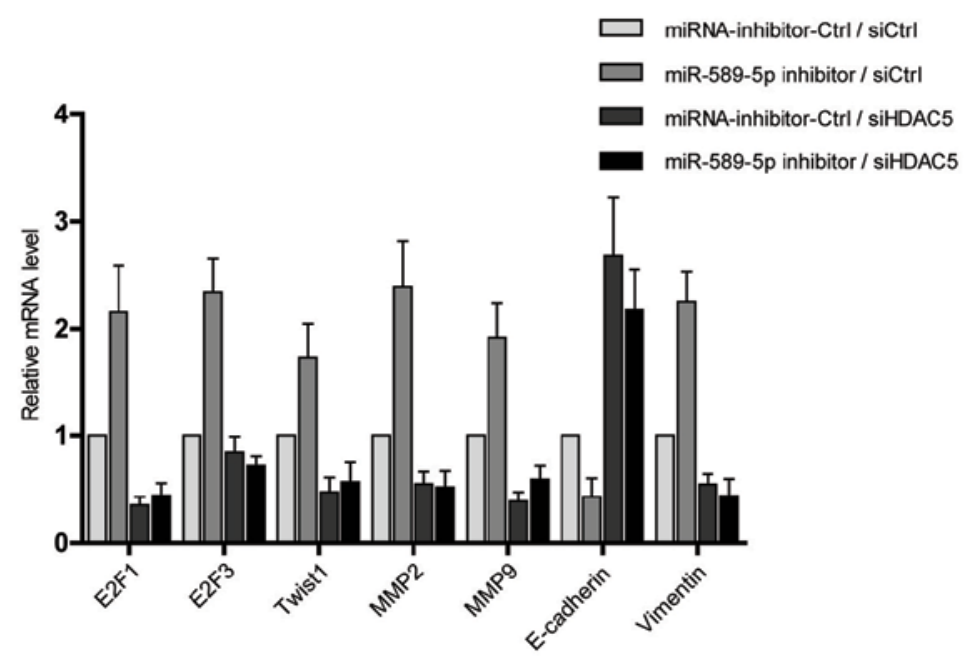

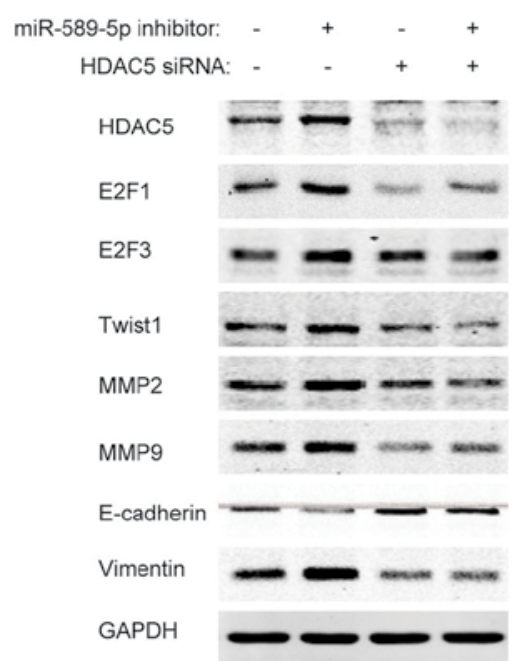

Figure 4. Upregulation of HDAC5 induces the expression of proliferation and EMT-related genes in NSCLC cells. (A) Heatmap representation of the relative mRNA levels for cell cycle gene clusters, EMT gene clusters, and tumor suppressor gene clusters in BEAS-2B cells with HDAC5 overexpression or A549 cells with HDAC5 knockdown. Red indicates that the gene was expressed at higher levels in treatment group compared with control; blue indicates that the gene was expressed at lower levels in treatment group compared with control. (B and C) Relative mRNA levels (B) and protein levels (C) of the indicated genes were confirmed by qPCR and western blotting in A549 cells with HDAC5 knockdown. "P<0.05. (D and E) Relative mRNA levels (D) and protein levels (E) of the indicated genes were confirmed by qPCR and western blotting in BEAS-2B cells with HDAC5 overexpression. " $\mathrm{P}<0.05$. (F and G) Relative mRNA levels (F) and protein levels $(\mathrm{G})$ of the indicated genes were examined by qPCR and western blotting in H1299 cells co-transfected with miR-589-5p inhibitor and HDAC5 siRNA. ${ }^{*} \mathrm{P}<0.05$. 
A

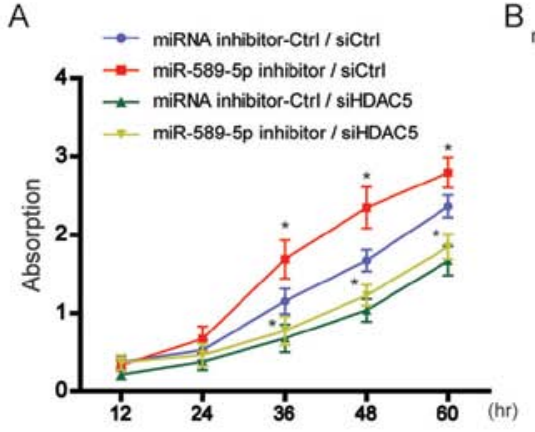

$B$ miR-589-5p inhibitor:

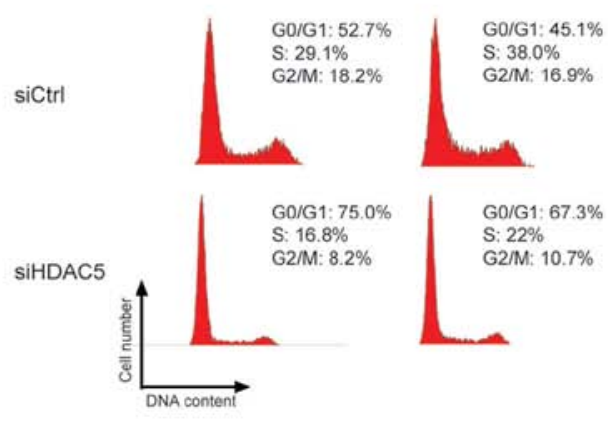

D

miR-589-5p inhibitor:

HDAC5 SiRNA.

Edu

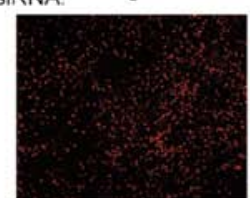

DAPI
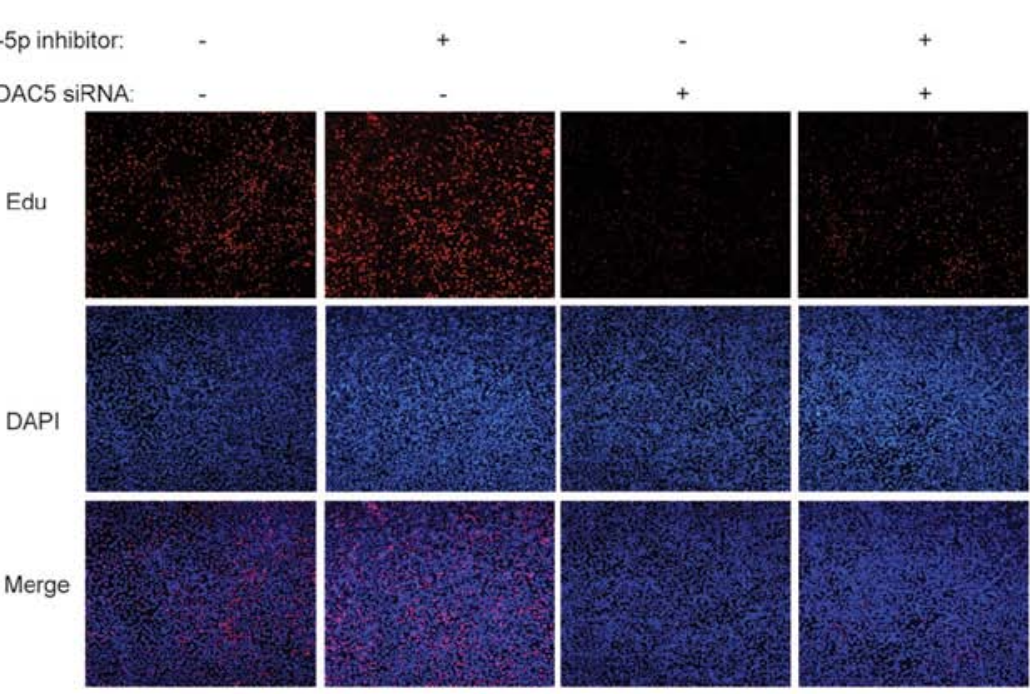

E
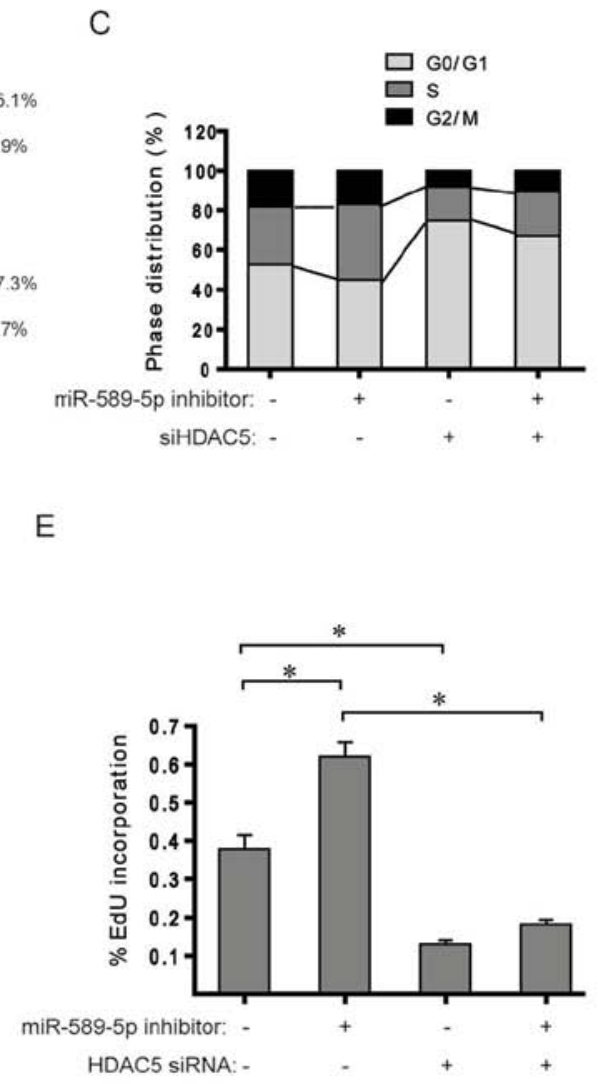

Figure 5. miR-589-5p/HDAC5 pathway regulates the proliferation of NSCLC cells. (A) At 12-60 h after co-transfection with miR-589-5p inhibitor and HDAC5 siRNA, the viability of H1299 cells were determined by the CCK-8 assay. ${ }^{*} \mathrm{P}<0.05$ (versus control group). (B) Cell cycle profiles of H1299 cells with the same transfection as (A) by flow cytometry. (C) The quantitative analysis for (B). "P<0.05. (D) DNA synthesis was measured by EdU incorporation assays in H1299 cells after the same transfection as (A). (E) Five visual fields under the fluorescent microscope were selected and calculated for each well. Counting was carried out by ImageJ software. ${ }^{*} \mathrm{P}<0.05$.

cells were treated with DNA methylation inhibitor, 5-Aza-2'deoxycytidine (5-Aza-dC), and the expression of HDAC5 was analyzed. The results showed that $>60 \%$ of the $\mathrm{CpGs}$ were demethylated in A549 cells treated with 5-Aza-dC (Fig. 3A). In line with this, miR-589-5p level was found to increase after 5-Aza-dC treatment (Fig. 3D and G). As expected, 5-Aza-dC observably suppressed the expression of HDAC5 in NSCLC cells, while miR-589-5p specific inhibitor weakened the suppression (Fig. 3E, F, H and I). Therefore, these findings suggest that silence of miR-589-5p in NSCLC is due to DNA hypermethylation.

Upregulation of HDAC5 induces the expression of proliferation and EMT-related genes in NSCLC cells. Since HDAC5 has been reported to play a crucial role in tumor progression and metastases, we examined the expression of several gene clusters associated with cell cycle and EMT by qPCR array. As shown in Fig. 4A, the data from qPCR array revealed that multiple cell cycle or EMT drivers, especially E2F1, E2F3, Twist1, MMP2, MMP9, and Vimentin, were robustly increased in the BEAS-2B cells with HDAC5 overexpression, but reduced in HDAC5-silenced NSCLC cells compared with control. Moreover, several tumor suppressor genes were also analyzed in the qPCR array, and their expression was repressed by HDAC 5 overexpression but activated after HDAC5 knockdown (Fig. 4A). The results of qPCR array were further confirmed by independent qPCR and western blotting (Fig. 4B-E). To determine the significance of miR-589-5p/HDAC5 pathway in regulating cell proliferation and EMT, the expressions of the above factors were examined in the NSCLC cells transfected with miR-589-5p inhibitor and HDAC5 siRNA. It was found that knockdown of HDAC5 completely reversed the promotive effect of miR-589-5p inhibitor on the expression of cell cycle or EMT drivers (Fig. 4F and G). Therefore, the evidence demonstrates that miR-589-5p/HDAC5 pathway plays a crucial role in regulating the cell cycle and EMT of NSCLC cells.

miR-589-5p/HDAC5 pathway regulates the proliferation of NSCLC cells. With regard to the data from Fig. 4, the effect of miR-589-5p/HDAC5 pathway on cell proliferation was explored in NSCLC cells. As shown in Fig. 5A, miR-589-5p inhibitor boosted cell proliferation and additional knockdown of HDAC5 completely suppressed the induced proliferation. Cell cycle analysis indicated that inhibiting miR-589-5p led to a significant increase in the ratio of $\mathrm{S}$ phase cells, but a decrease after transfection with HDAC5 siRNA (Fig. 5B 
A

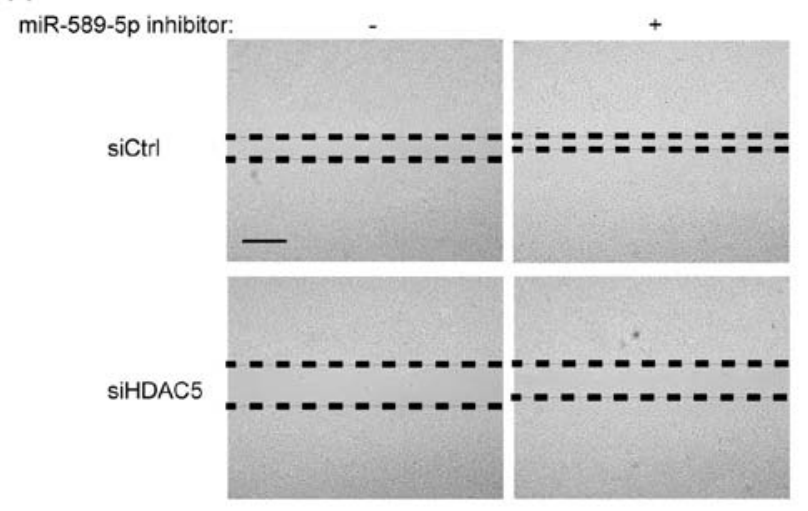

C

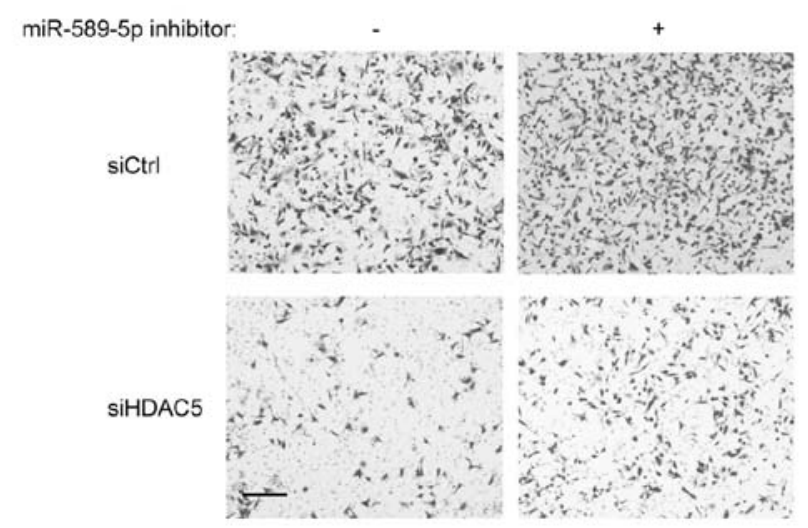

B

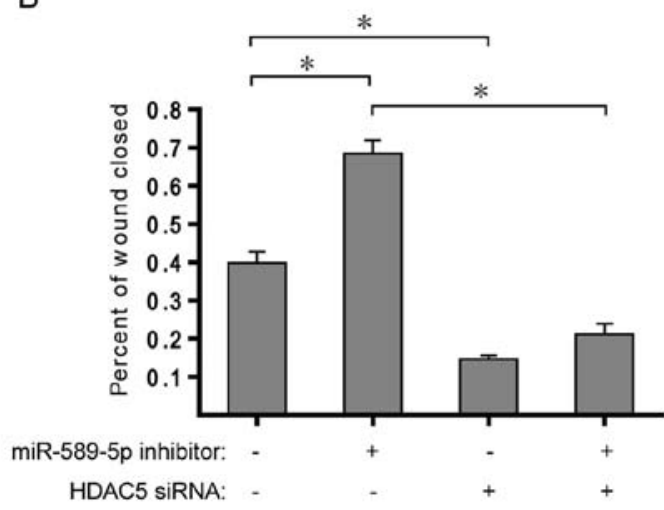

D

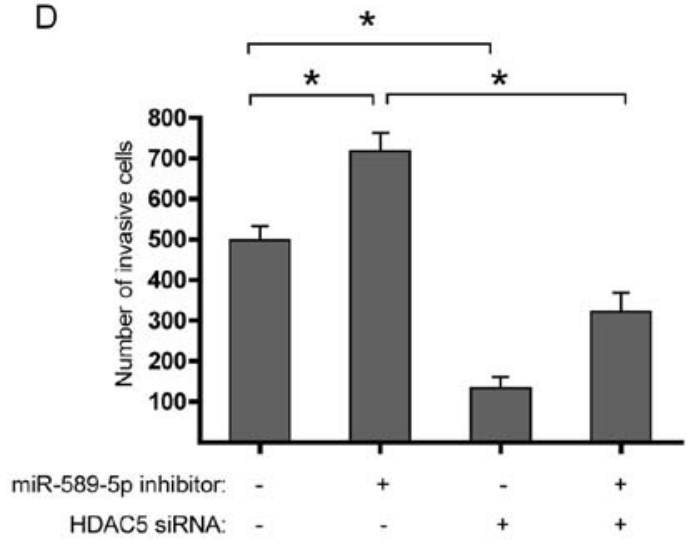

Figure 6. miR-589-5p/HDAC5 pathway modulates the migration and invasion of NSCLC cells. (A) Representative images from scratch assays with H1299 cells after co-transfection with miR-589-5p inhibitor and HDAC5 siRNA. Scale bar, $200 \mu \mathrm{m}$ (B) Percentage wound closure $48 \mathrm{~h}$ after the indicated transfection. ${ }^{*} \mathrm{P}<0.05$. (C) Representative images of $\mathrm{H} 1299$ cells with the same transfection as (A) penetrating the Matrigel in invasion assays. Scale bar, $200 \mu \mathrm{m}$. (D) Numbers of invasive cells with the indicated transfection for $48 \mathrm{~h}$. ${ }^{*} \mathrm{P}<0.05$.

and C). Similarly, EdU incorporation assay revealed that the percentage of cells with incorporated EdU was significantly increased when treated with miR-589-5p inhibitor; however, knockdown of HDAC5 impaired the effect (Fig. 5D and E). The above results indicate a regulatory role of miR-589-5p/HDAC5 pathway in the proliferation of NSCLC cells.

miR-589-5p/HDAC5 pathway modulates the migration and invasion of NSCLC cells. Considering the upregulation of EMT-related genes induced by HDAC5 overexpression, it is worth studying the role of miR-589-5p/HDAC5 pathway in the migration and invasion of NSCLC. It was found that suppression of miR-589-5p markedly strengthened the migratory and invasive capabilities of NSCLC cells, whereas deprivation of HDAC5 determined the reverse in the malignant phenotypes (Fig. 6). Taken together, these findings suggest that deregulation of miR-589-5p/HDAC5 pathway displays a critical role in the migration and invasion of NSCLC.

Dysregulation of miR-589-5p/HDAC5 pathway promotes NSCLC tumorigenesis. To further determine the role of miR-589-5p/HDAC5 pathway in tumorigenesis, A549 cells with stable overexpression of miR-589-5p and HDAC5 were constructed and then examined by colony formation and xenograft assays. As shown in Fig. 7A and B, overexpression of miR-589-5p resulted in the reduction of anchorage-independent colony formation in soft agar in A549 cells, but additional exogenous HDAC5 rescued the reduction. Xenograft assay demonstrated that overexpression of miR-589-5p significantly decreased A549 xenograft growth in nude mice while simultaneous HDAC5 overexpression partially retrieved the growth (Fig. 7C-E). Taken together, these data indicate a crucial role of miR-589-5p/HDAC5 pathway in NSCLC tumorigenicity in vitro and in vivo.

\section{Discussion}

Over the past decade, epigenetic dysregulation including DNA methylation patterns, histone modifications and microRNA regulation has been reported to result in the initiation and progression of lung cancer $(13,14)$. As the most studied epigenetic regulatory mechanism, DNA methylation plays a crucial role in suppressing gene expression and maintaining normal epigenetic regulatory processes (15). However, in cancer cells, CpG islands located at the promoters of tumor suppression genes are highly methylated leading to transcriptional repression (16). Similarly, as an important tumor suppressor miRNA, miR-589-5p was found drastically decreased in NSCLC compared with normal lung tissues. Further analysis indicated that miR-589-5p silence was relevant for a strong 
A

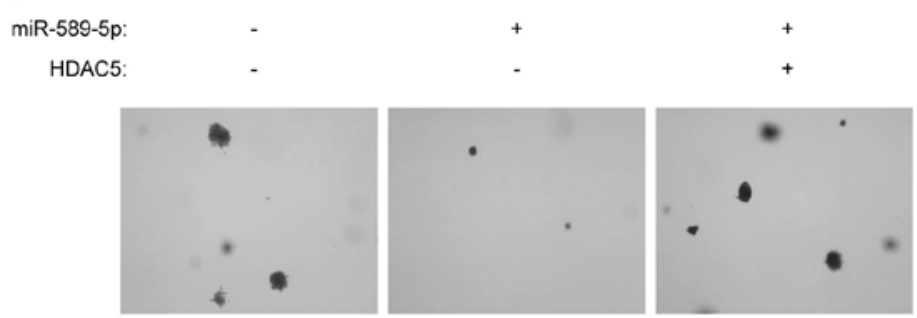

B

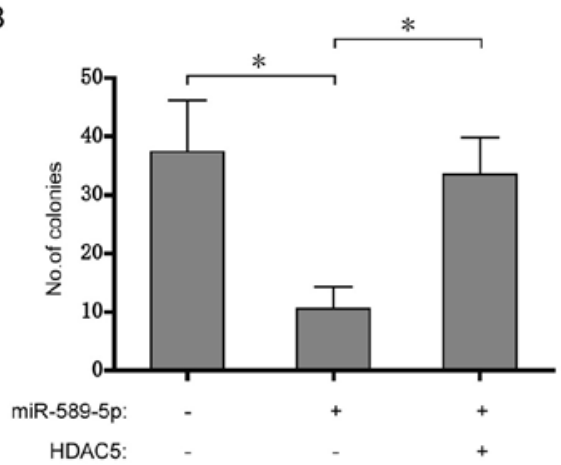

C

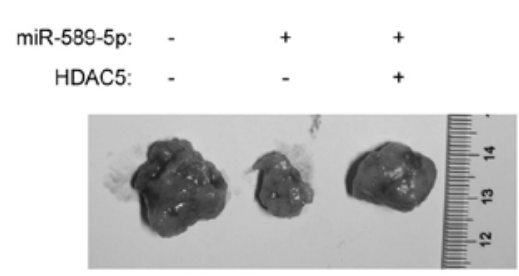

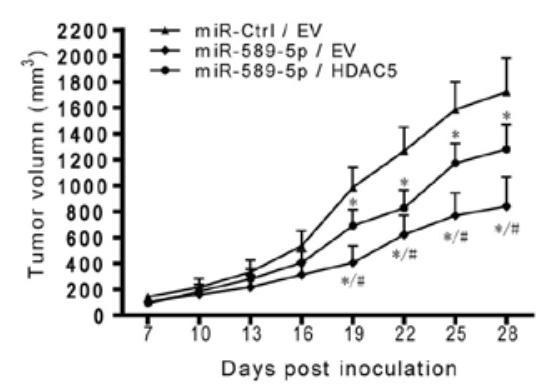

$\mathrm{E}$

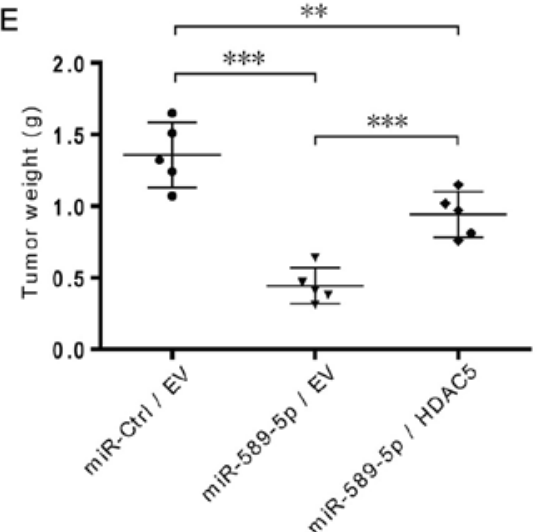

Figure 7. Dysregulation of miR-589-5p/HDAC5 pathway promotes NSCLC tumorigenesis. (A) Representative images of colony formation in A549 cells with indicated stable transfection. (B) Quantification of colony formation in (A). "P<0.05. (C) Images of tumors formed by A549 cells with indicated stable transfection. (D) Time-course of tumor volume progression, as determined by caliper measurements. ${ }^{~} \mathrm{P}<0.05$. (E) Tumor weights as measured when mice were sacrificed on day $28 .{ }^{*} \mathrm{P}<0.05 ;{ }^{* *} \mathrm{P}<0.01 ;{ }^{* * *} \mathrm{P}<0.001$.

gain of DNA methylation in the miR-589 gene promoter. Consistently, treatment with methylation inhibitor (5-Aza-dC) achieved restoration of miR-589-5p expression in NSCLC cells. Although the mechanisms underlying the promoter hypermethylation of the miR-589 gene were not the focus of the present study, previous studies described some putative factors involved in deregulated DNA methylation in lung cancer. DNA methyltransferases (DNMTs) are responsible for producing methylated $\mathrm{CpG}$ and aberrant DNMT expression is implicated in the pathogenesis of lung cancer $(17,18)$. Upregulation of DNMTs silences different tumor suppressors by promoter hypermethylation in lung cancer (18). Therefore, DNA methylation has become a therapeutic target which can be disrupted by DNMT inhibitors, such as decitabine and 5-Aza-dC (19).

In addition to DNA methylation, histone modifications including acetylation, phosphorylation, and methylation also contributes to epigenetic alterations in lung cancer. Although the roles of HDAC family in tumorigenesis have not been fully understood, HDAC inhibitors are emerging as novel anticancer agents due to their ability to kill cancer cells by inducing cell cycle arrest, autophagy, and apoptosis (20-22). Here, accompanied with decreased miR-589-5p level, a higher expression of HDAC5 was observed in NSCLC compared with normal lung tissues. Further evidence showed that miR-589-5p significantly repressed the expression of HDAC 5 by targeting the 3 'UTR of HDAC5 mRNA in NSCLC cells. As an important epigenetic modulator, HDAC5 promotes cell growth, migra- tion, and invasion of breast cancer and high levels of HDAC5 are closely related with poor survival in human brain cancer patients $(23,24)$. With regard to miR-589-5p/HDAC5 pathway found in the study, it is reasonable to hypothesize that HDAC5 may function as a critical target of deregulated miR-589-5p, which promotes NSCLC aggressiveness. As expected, the results demonstrated that HDAC5 mediated the malignant phenotypes including proliferation, migration, invasion, and tumorigenicity induced by silence of miR-589-5p in NSCLC cells.

HDAC5 has a broad range of downstream genes, including some important transcription factors or modulators, such as p53, CDK, and DLL4 $(25,26)$. The diverse functions of HDAC5 depend on its downstream genes which regulate different cellular biological processes. For this reason, we extended our studies to examine the expression profiling of gene clusters associated with cell cycle and EMT by qPCR array. The screening results showed that the cell cycle and EMT drivers were widely downregulated after depletion of HDAC 5 but upregulated by HDAC5 overexpression. Specially, cell cycle drivers, such as E2F1 and E2F3, as well as EMT-inducing transcription factors (EMT-TFs) like Twist1 were markedly induced after HDAC5 overexpression. In lung cancer, abnormality in E2F1 expression has been described and associated with poor patient survival (27). As a well-known transcription factor, E2F1 transactivates various downstream effectors, such as ribonucleotide reductase $\mathrm{m} 2$ (RRM2) and thymidylate synthase (TS), which lead to tumor 
proliferation in NSCLC $(28,29)$. Of note, it was found that E2F1 could activate the transcription of DNMT3A which specially resulted in an increased methylation level and suppression of tumor suppressor genes (TSGs) (30). Therefore, inhibition of miR-589-5p by DNA methylation may be maintained via a positive feedback, miR-589-5p/HDAC5/E2F1/DNMT3A loop, in NSCLC. As a well-documented member of EMT-TFs, Twist1 has been reported to induce epithelial-mesenchymal transition and metastasis in NSCLC (31). In addition, aberrant expression of Twist1 determines lung cancer chemoresistance and poor survival (32). Consistent with upregulated Twist1, Increased mesenchymal marker (Vimentin) and decreased epithelial marker (E-cadherin) indicated HDAC5-induced EMT.

However, in contrast with above gene clusters, the expressions of TSGs were negatively regulated by HDAC5 in NSCLC or lung epithelial cells. Among these TSGs, p21 and PTEN were significantly suppressed by HDAC5. Decreased PTEN expression level, which occurs in up to $70 \%$ NSCLC patients, is associated to lower survival (33). Loss of PTEN expression was found to accelerate the EMT and development of lung cancer by activating PI3K/AKT pathway $(34,35)$. Thus, the expression profiles of HDAC5 downstream effectors need to be deeply investigated to understand the roles of HDAC5 in lung cancer.

Therefore, we analyzed the effects of miR-589-5p/ HDAC5 pathway on malignant phenotypes, viz proliferation, migration, invasion, and tumorigenicity. Consistent with the above results, HDAC5 inhibition conferred the function of miR-589-5p as a tumor suppressor in NSCLC cells. In hepatocellular carcinoma, knockdown of HDAC5 inhibits HCC cell proliferation and tumorigenicity in nude mice (25). Besides, suppression of HDAC5 sensitizes glioma cells to chemotherapeutics by preventing EMT (36). However, overexpression of HDAC5 inhibits tumor cell growth and induces apoptosis in the osteosarcoma U2OS cells (37). Thus, distinct behavior of HDAC5 towards cell fate decisions in different cancer types are worth studying in the future.

In conclusion, the present study demonstrates a poor prognosis in NSCLC patients with high HDAC5 expression and a negative correlation between miR-589-5p and HDAC5 in NSCLC specimens. Further analysis showed that HDAC5 is directly targeted by miR-589-5p, and hypermethylation-mediated silence of miR-589-5p results in the aberrant high expression of HDAC5 in NSCLC cells. Moreover, miR-589-5p/HDAC5 pathway regulates the expression of cell cycle and EMT-related gene clusters and consequently determines the capabilities of proliferation, migration, invasion, and tumorigenicity in NSCLC cells. Therefore, abnormal miR-589-5p/HDAC5 pathway caused by hypermethylation contributes to the aggressiveness of NSCLC.

\section{References}

1. Álvarez-Fernández C and Esteban-González E: Current status of EGFR/ErbB inhibitors in non-small cell lung carcinoma. Med Clin (Barc) 146 (Suppl 1): 2-6, 2016 (In Spanish).

2. Juergens RA, Wrangle J, Vendetti FP, Murphy SC, Zhao M, Coleman B, Sebree R, Rodgers K, Hooker CM, Franco N, et al: Combination epigenetic therapy has efficacy in patients with refractory advanced non-small cell lung cancer. Cancer Discov 1: 598-607, 2011.
3. Martin M, Kettmann R and Dequiedt F: Class IIa histone deacetylases: Conducting development and differentiation. Int J Dev Biol 53: 291-301, 2009.

4. Mottet D, Pirotte S, Lamour V, Hagedorn M, Javerzat S, Bikfalvi A, Bellahcène $\mathrm{A}$, Verdin $\mathrm{E}$ and Castronovo V: HDAC4 represses p21 (WAF1/Cip1) expression in human cancer cells through a Sp1-dependent, p53-independent mechanism. Oncogene 28: 243-256, 2009.

5. Feng GW, Dong LD, Shang WJ, Pang XL, Li JF, Liu L and Wang Y: HDAC5 promotes cell proliferation in human hepatocellular carcinoma by up-regulating Six 1 expression. Eur Rev Med Pharmacol Sci 18: 811-816, 2014.

6. Cacan E: Histone deacetylase-1-mediated suppression of FAS in chemoresistant ovarian cancer cells. Anticancer Res 36: 2819-2826, 2016.

7. Liu J, Gu J, Feng Z, Yang Y, Zhu N, Lu W and Qi F: Both HDAC5 and HDAC6 are required for the proliferation and metastasis of melanoma cells. J Transl Med 14: 7, 2016.

8. Wang L, Li H, Ren Y, Zou S, Fang W, Jiang X, Jia L, Li M, Liu X, Yuan X, et al: Targeting HDAC with a novel inhibitor effectively reverses paclitaxel resistance in non-small cell lung cancer via multiple mechanisms. Cell Death Dis 7: e2063, 2016.

9. Zhang K, Zhang H, Zhou X, Tang WB, Xiao L, Liu YH, Liu H, Peng YM, Sun L and Liu FY: miRNA589 regulates epithelialmesenchymal transition in human peritoneal mesothelial cells. J Biomed Biotechnol 2012: 673096, 2012.

10. Li W, Wang W, Ding M, Zheng X, Ma S and Wang X: MiR-1244 sensitizes the resistance of non-small cell lung cancer A549 cell to cisplatin. Cancer Cell Int 16: 30, 2016.

11. Zhang X, Jiang P, Shuai L, Chen K, Li Z, Zhang Y, Jiang Y and Li X: miR-589-5p inhibits MAP3K8 and suppresses CD90 ${ }^{+}$ cancer stem cells in hepatocellular carcinoma. J Exp Clin Cancer Res 35: 176, 2016.

12. Dávalos-Salas M, Furlan-Magaril M, González-Buendía E, Valdes-Quezada C, Ayala-Ortega E and Recillas-Targa F: Gain of DNA methylation is enhanced in the absence of CTCF at the human retinoblastoma gene promoter. BMC Cancer 11: 232, 2011.

13. Brzeziańska E, Dutkowska A and Antczak A: The significance of epigenetic alterations in lung carcinogenesis. Mol Biol Rep 40: 309-325, 2013.

14. Balgkouranidou I, Liloglou T and Lianidou ES: Lung cancer epigenetics: Emerging biomarkers. Biomarkers Med 7: 49-58, 2013.

15. Miranda TB and Jones PA: DNA methylation: The nuts and bolts of repression. J Cell Physiol 213: 384-390, 2007.

16. Mehta A, Dobersch S, Romero-Olmedo AJ and Barreto G: Epigenetics in lung cancer diagnosis and therapy. Cancer Metastasis Rev 34: 229-241, 2015.

17. Patel K, Dickson J, Din S, Macleod K, Jodrell D and Ramsahoye B: Targeting of 5-aza-2'-deoxycytidine residues by chromatinassociated DNMT1 induces proteasomal degradation of the free enzyme. Nucleic Acids Res 38: 4313-4324, 2010.

18. Damiani LA, Yingling CM, Leng S, Romo PE, Nakamura J and Belinsky SA: Carcinogen-induced gene promoter hypermethylation is mediated by DNMT1 and causal for transformation of immortalized bronchial epithelial cells. Cancer Res 68: 9005-9014, 2008.

19. Forde PM, Brahmer JR and Kelly RJ: New strategies in lung cancer: Epigenetic therapy for non-small cell lung cancer. Clin Cancer Res 20: 2244-2248, 2014.

20. Liu ZH, Li J, Xia J, Jiang R, Zuo GW, Li XP, Chen Y, Xiong W and Chen DL: Ginsenoside 20 (s)-Rh2 as potent natural histone deacetylase inhibitors suppressing the growth of human leukemia cells. Chem Biol Interact 242: 227-234, 2015.

21. Zhang J, Ng S, Wang J, Zhou J, Tan SH, Yang N, Lin Q, Xia D and Shen HM: Histone deacetylase inhibitors induce autophagy through FOXO1-dependent pathways. Autophagy 11: 629-642, 2015.

22. Jang SM, Kang EJ, Kim JW, Kim CH, An JH and Choi KH: Transcription factor Sox4 is required for PUMA-mediated apoptosis induced by histone deacetylase inhibitor, TSA. Biochem Biophys Res Commun 438: 445-451, 2013.

23. Li A, Liu Z, Li M, Zhou S, Xu Y, Xiao Y and Yang W: HDAC5, a potential therapeutic target and prognostic biomarker, promotes proliferation, invasion and migration in human breast cancer. Oncotarget 7: 37966-37978, 2016.

24. Milde T, Oehme I, Korshunov A, Kopp-Schneider A, Remke M, Northcott P, Deubzer HE, Lodrini M, Taylor MD, von Deimling A, et al: HDAC5 and HDAC9 in medulloblastoma: Novel markers for risk stratification and role in tumor cell growth. Clin Cancer Res 16: 3240-3252, 2010. 
25. Fan J, Lou B, Chen W, Zhang J, Lin S, Lv FF and Chen Y: Downregulation of HDAC 5 inhibits growth of human hepatocellular carcinoma by induction of apoptosis and cell cycle arrest. Tumour Biol 35: 11523-11532, 2014.

26. He P, Liang J, Shao T, Guo Y, Hou Y and Li Y: HDAC5 promotes colorectal cancer cell proliferation by up-regulating DLL4 expression. Int J Clin Exp Med 8: 6510-6516, 2015.

27. Gorgoulis VG, Zacharatos P, Mariatos G, Kotsinas A, Bouda M, Kletsas D, Asimacopoulos PJ, Agnantis N, Kittas C and Papavassiliou AG: Transcription factor E2F-1 acts as a growthpromoting factor and is associated with adverse prognosis in non-small cell lung carcinomas. J Pathol 198: 142-156, 2002.

28. Grossi F, Dal Bello MG, Salvi S, Puzone R, Pfeffer U, Fontana V, Alama A, Rijavec E, Barletta G, Genova C, et al: Expression of ribonucleotide reductase subunit-2 and thymidylate synthase correlates with poor prognosis in patients with resected stages I-III non-small cell lung cancer. Dis Markers 2015: 302649, 2015.

29. Noro R, Miyanaga A, Minegishi Y, Okano T, Seike M, Soeno C, Kataoka K, Matsuda K, Yoshimura A and Gemma A: Histone deacetylase inhibitor enhances sensitivity of non-small-cell lung cancer cells to 5-FU/S-1 via down-regulation of thymidylate synthase expression and up-regulation of $\mathrm{p} 21$ (waf1/cip1) expression. Cancer Sci 101: 1424-1430, 2010.

30. Tang YA, Lin RK, Tsai YT, Hsu HS, Yang YC, Chen CY and Wang YC: MDM2 overexpression deregulates the transcriptional control of RB/E2F leading to DNA methyltransferase 3A overexpression in lung cancer. Clin Cancer Res 18: 4325-4333, 2012.

31. $\mathrm{Li} \mathrm{L}$ and $\mathrm{Wu} \mathrm{D}: \mathrm{miR}-32$ inhibits proliferation, epithelialmesenchymal transition, and metastasis by targeting TWIST1 in non-small-cell lung cancer cells. Onco Targets Ther 9: 1489-1498, 2016.
32. Ávila-Moreno F, Armas-López L, Álvarez-Moran AM, López-Bujanda Z, Ortiz-Quintero B, Hidalgo-Miranda A, Urrea-Ramírez F, Rivera-Rosales RM, Vázquez-Manríquez E, Peña-Mirabal E, et al: Overexpression of MEOX2 and TWIST1 is associated with $\mathrm{H} 3 \mathrm{~K} 27 \mathrm{me} 3$ levels and determines lung cancer chemoresistance and prognosis. PLoS One 9: e114104, 2014.

33. Pérez-Ramírez C,Cañadas-Garre M, Molina MA, Faus-Dáder MJ and Calleja-Hernández MA: PTEN and PI3K/AKT in non-small-cell lung cancer. Pharmacogenomics 16: 1843-1862, 2015.

34. Li J, Yang S, Yan W, Yang J, Qin YJ, Lin XL, Xie RY, Wang SC, Jin W, Gao F, et al: MicroRNA-19 triggers epithelial-mesenchymal transition of lung cancer cells accompanied by growth inhibition. Lab Invest 95: 1056-1070, 2015.

35. Yun F, Jia Y, Li X, Yuan L, Sun Q, Yu H, Shi L and Yuan H: Clinicopathological significance of PTEN and PI3K/AKT signal transduction pathway in non-small cell lung cancer. Int J Clin Exp Pathol 6: 2112-2120, 2013.

36. Liu Q, Sun Y, Zheng JM, Yan XL, Chen HM, Chen JK and Huang HQ: Formononetin sensitizes glioma cells to doxorubicin through preventing EMT via inhibition of histone deacetylase 5. Int J Clin Exp Pathol 8: 6434-6441, 2015.

37. Huang Y, Tan M, Gosink M, Wang KK and Sun Y: Histone deacetylase 5 is not a p53 target gene, but its overexpression inhibits tumor cell growth and induces apoptosis. Cancer Res 62: 2913-2922, 2002. 